\title{
Adaptação e validação da escala de autoeficácia no uso de preservativo em uma amostra brasileira
}

\author{
Josemberg Moura de Andrade \\ Universidade de Brasília, DF, Brasil \\ Kaline da Silva Lima \\ Valdiney Veloso Gouveia \\ Hemerson Fillipy Silva Sales \\ Élida de Farias Melo \\ Vivianne Freitas Oliveira Asfora \\ Universidade Federal da Paraíba, PB, Brasil
}

\begin{abstract}
Resumo
A autoeficácia no uso de preservativos está relacionada à saúde, principalmente na prevenção de infecções sexualmente transmissíveis. Objetivou-se adaptar a Escala de Autoeficácia no Uso de Preservativos para o contexto brasileiro. Para isso, foram realizados dois estudos a partir de um questionário online. O Estudo 1 contou com 332 participantes da população geral, a maioria mulheres $(63,6 \%)$, com média de idade de 25,2 anos ( $D P=7,4)$. A análise fatorial exploratória identificou quatro fatores: Habilidade, Assertividade, Prazer e Drogas e Doenças sexualmente transmissíveis, explicando 66,8\% da variância. O Estudo 2 contou com 907 participantes, com média de idade de 23,6 anos ( $\mathrm{DP}=6,1)$. A análise fatorial confirmatória apresentou os seguintes indicadores de ajuste: $\mathrm{GFI}=0,93$; $\mathrm{CFI}=0,91$; RMSEA=0,08 (IC90\%=0,07-0,09). Os resultados comprovam a adequação da medida, reunindo evidências de validade. Discute-se a importância desta medida em pesquisas na área de saúde.
\end{abstract}

Palavras-chave: Autoeficácia; Prevenção; Validade de construto; Comportamento sexual.

\section{Adaptation and validation of the condom use self efficacy scale in a brazilian sample}

\begin{abstract}
Self-efficacy in the use of condoms is related to health, especially in the prevention of sexually transmitted infections. This research sought to adapt the Condom Use Self-Efficacy Scale for the Brazil. For this, two studies were conducted through an online questionnaire. Study 1 had 332 participants from the general population; the majority of women (63.6\%), with a mean age of 25.2 years $(\mathrm{SD}=7.4)$. The exploratory factor analysis identified four factors: Ability, Assertiveness, Pleasure and Drugs and Sexually Transmitted Diseases, explaining 66.8\% of the variance. Study 2 had 907 participants, with a mean age of 23.6 years $(\mathrm{SD}=6.1)$. Confirmatory factor analysis corroborated the structure found: $\mathrm{GFI}=0.93$; $\mathrm{CFI}=0.91$; RMSEA $=0.08$ (IC 90\% $=0.07-0.09$ ). The results proved the adequacy of the measure, gathering evidence of validity. The importance of this measure in health research is discussed.
\end{abstract}

Keywords: Self-efficacy; Prevention; Construct validity; Sexual behavior.

\section{Adaptación y validación de la escala de autoeficacia en el uso de preservativo en una muestra brasileña}

\section{Resumen}

La autoeficacia en el uso de preservativos está relacionada con la salud, principalmente en la prevención de infecciones de transmisión sexual. Esta investigación buscó adaptar la Escala de Autoeficacia en el uso de preservativos para Brasil. Para ello, se realizaron dos estudios a través de un cuestionario en línea. El Estudio 1 contó con 332 participantes de la población general, la mayoría mujeres $(63,6 \%)$, con una media de edad de 25,2 años $(\mathrm{DP}=7,4)$. El análisis factorial exploratorio identificó cuatro factores: Habilidad, Asertividad, Placer y Drogas y Enfermedades de transmisión sexual, explicando el 66,8\% de la varianza. El Estudio 2 contó con 907 participantes, con una media de edad de 23,6 años $(\mathrm{DP}=6,1)$. El análisis factorial confirmatorio corroboró la estructura encontrada: $\mathrm{GFI}=0,93 ; \mathrm{CFI}=0,91 ; \mathrm{RMSEA}=0,08(\mathrm{IC} 90 \%=0,07-0,09)$. Los resultados comprobaron la adecuación de la medida, reuniendo evidencias de validez. Se discute la importancia de esta medida en investigaciones en el área de salud. 


\section{Introdução}

A não adesão ao uso de preservativos ainda é uma realidade observada, principalmente, em adolescentes e em adultos jovens de ambos os sexos. Essa população é mais vulnerável à gravidez indesejada e/ou precoce e às infecções sexualmente transmissíveis (ISTs), como o HIV/aids, que ainda é considerado um importante problema de saúde pública a nível global (Lima et al., 2016).

No caso do Brasil, de acordo com o último Boletim Epidemiológico divulgado pelo Ministério da Saúde (2016), desde o início da epidemia até junho de 2016, foram notificados no país 842.710 casos de HIV. A maior concentração dos casos acomete a população com idades entre 25 e 39 anos. No que se refere às regiões, o sudeste $(53 \%)$ e sul $(20,1 \%)$ apresentam maiores quantitativos de infectados, seguido do nordeste $(15,1 \%)$, centro-oeste $(6 \%)$ e norte $(5,9 \%)$.

No ano de 2015, a principal forma de transmissão da aids em indivíduos maiores de 13 anos ocorreu via sexual, tanto em homens $(95,3 \%)$ quanto em mulheres $(97,1 \%)$. Na população masculina, verificou-se um predomínio da categoria de orientação heterossexual. Entretanto, há uma tendência de aumento na proporção de casos em homens que fazem sexo com outros homens (HSH) na última década, representando 45,4\% dos casos em 2015 (Ministério de Saúde, 2016).

Nos últimos anos, a prevenção efetiva ao HIV se apresenta por meio de uma combinação de várias estratégias de intervenções comportamentais, biomédicas e estruturais, com grandes expectativas nas chamadas "novas tecnologias de prevenção" (circuncisão, microbicidas, profilaxia pós-exposição - PEP, profilaxia pré-exposição - PrEP). Entre as estratégias comportamentais, inclui-se o soroposicionamento, soro-adaptação e outras modalidades de acordo com os parceiros sexuais (Cohen, Liu, Bernstein, \& Philip, 2013; Dourado, MacCarthy, Reddy, Calazans, \& Gruskin, 2015). Apesar do avanço considerável dos estudos interventivos e preventivos, o que se observa é um aumento do HIV em jovens no Brasil, em contrapartida, uma preocupante tendência de redução do uso regular do preservativo (Dourado et al., 2015).

O preservativo foi, e continua sendo, a principal aposta no campo da prevenção desde o início das políticas relativas à aids no Brasil (Pinheiro, Calazans, \& Ayres, 2013). Estudos recentes investigaram possíveis causas para o não uso de preservativos pelos jovens brasileiros, os quais verificaram, dentre os motivos principais a preferência por métodos anticoncepcionais, confiança no parceiro, ou o simples fato de não gostar (Longo, 2013; Pereira et al., 2014; Ribeiro, Silva, \& Saldanha, 2011; Tronco \& Dell'Aglio, 2012).

As elevadas taxas de prevalência de doenças sexualmente transmissíveis, especialmente o HIV/aids, têm direcionado ao desenvolvimento de modelos teóricos e instrumentos de medida que buscam conhecer os comportamentos individuais de risco sexual (Santos, Ferreira, Duarte, \& Ferreira, 2017). Nessa perspectiva, um importante construto a ser estudado no âmbito da saúde e que tem se mostrado fortemente associado ao comportamento frente ao uso do preservativo é o de autoeficácia.

Segundo Myers (2000), a autoeficácia é a medida de quão competente uma pessoa se sente para fazer alguma coisa, envolvendo o controle sobre seus resultados, conceito que ganhou reconhecimento como uma ferramenta para facilitar a mudança comportamental positiva. O termo é derivado da Teoria da Aprendizagem Social de Bandura (1994), teórico que propôs a autoeficácia como um mediador importante de comportamento e definiu o conceito como "um julgamento da própria capacidade para realizar um determinado nível de desempenho" (Bandura, 1986, p. 390).

Na vida cotidiana, a autoeficácia leva o indivíduo a fixar objetivos desafiadores e a persistir quando se defronta com dificuldades. Quando surgem os problemas, o indivíduo pode manter a calma e procurar soluções. Essa habilidade está relacionada às realizações alcançadas, à autoconfiança e à autoestima. No entanto, nem sempre a autoeficácia dispõe de pontos positivos ou de benefícios. O otimismo ilusório ou irrealista, que pode ser gerado pela autoeficácia, aumenta a vulnerabilidade do indivíduo (Myers, 2000).

A partir da perspectiva teórica proposta por Bandura (1986), enfatiza-se o modelo de autoeficácia como uma explicação de comportamentos sexuais com risco de IST, apoiando-se em um modelo conceitual que articula a teoria sociocognitiva e a autoeficácia percebida ao controle de atividades sexuais. A concepção de Bandura entende que o comportamento é mediado por um processo de avaliação cognitiva em que as pessoas integram conhecimentos, expectativas de resultado, estados emocionais, influências sociais e experiências passadas, criando assim uma estimativa de sua capacidade de lidar com uma determinada situação (Wulfert \& Wan, 1993).

Como se percebe, a Teoria da Autoeficácia influencia de forma considerável os estudos de comportamentos relacionados à saúde, incluindo a prevenção da transmissão sexual do HIV. Por isso, no presente estudo, a autoeficácia no uso de preservativos 
parte do modelo de mensuração proposto por Brafford e Beck (1991) que foi desenvolvido a partir de estudos realizados com a Condom Use Self-Efficacy Scale (CUSES). Essa medida foi originalmente construída para a população norte-americana e tem sido bastante utilizada em diversos países como Índia (Asante \& Doku, 2010), Étiópia (Shaweno \& Tekletsadik, 2013) e Portugal (Santos et al., 2017). O artigo de Brafford e Beck (1991) que propõe a referida escala possui mais de 340 citações de estudos conduzidos em diversos países, indicando a sua relevância prática e teórica. Ressalta-se, no entanto, que no Brasil não se encontra qualquer instrumento que permita avaliar a autoeficácia dos indivíduos no que se refere ao uso do preservativo.

O processo de construção dos itens da CUSES foi derivado de três fontes: literatura anterior, contribuição de especialistas e estudantes universitários. Baseouse em escalas previamente desenvolvidas como a Attitude Toward the Condom Scale (ATC) e Levinson's

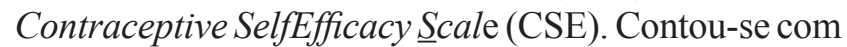
cinco especialistas que trabalhavam com estudantes universitários no campo da sexualidade e 183 estudantes que responderam a um questionário aberto acerca dos fatores que eles acreditavam influenciar o uso de preservativos. A primeira versão da escala resultou em 28 itens específicos de autoeficácia que descrevem sentimentos de um indivíduo acerca de sua confiança sobre a possibilidade de comprar preservativos, colocá-los e tirá-los e, também, de negociar o seu uso com um novo parceiro sexual (Brafford \& Beck, 1991). Essa escala foi aplicada a uma amostra de 768 estudantes universitários, verificando-se uma adequada confiabilidade, dado um coeficiente alfa de Cronbach igual a 0,91 , uma correlação teste-reteste de 0,81 e boa correlação com a Attitude Toward the Condom Scale $(r=0,51)$ e com a Contraceptive Self-Efficacy Scale for Women $(r=0,55)$.

Uma versão reduzida foi proposta por Brien, Thombs, Mahoney e Wallnau (1994). Essa versão apresentou uma estrutura multidimensional a qual foi composta por 15 itens organizados em quatro dimensões designadas: mecanismos, desaprovação do parceiro, assertividade e intoxicantes. Os valores de alfa de Cronbach variaram entre 0,78 (mecanismos) e 0,82 (assertividade e intoxicantes). Embora tal estrutura tenha se mostrado consistente, algumas alterações foram observadas em estudos posteriores (Asante \& Doku, 2010; Santos et al., 2017).

Para esta pesquisa optou-se por considerar a estrutura proposta por Asante e Doku (2010), visto o fato desta ter apresentado melhores resultados de fidedignidade. Esses autores propuseram uma estrutura multifatorial de 14 itens e quatro fato- res (Habilidade; Assertividade; Prazer e Drogas; e Doenças Sexualmente Transmissíveis). O primeiro fator - Habilidade - está relacionado com a habilidade mecânica no uso do preservativo. Esta dimensão é indicativa de autoeficácia relacionada com as habilidades multifacetadas no uso do preservativo direcionado à própria pessoa e ao parceiro. $\mathrm{O}$ segundo fator - Assertividade - está relacionado à autoeficácia no que diz respeito às habilidades de negociação e capacidade para convencer um(a) parceiro(a) a usar o preservativo. Tal aspecto é relacionado ao aumento da consciência sobre os riscos associados com a sua não utilização. O terceiro fator - Prazer e Drogas - indica a capacidade de utilizar preservativos sob a influência de álcool ou drogas sem sentir uma redução na sensação sexual. Por fim, o último fator - DSTs - apresenta itens que envolvem o receio do(a) respondente de que o(a) parceiro(a) pense que ela(a) possui alguma doença sexualmente transmissível. Este fator está intimamente ligado à desaprovação do(a) parceiro(a) (Asante \& Doku, 2010). Os valores alfas de Cronbach dessas dimensões variaram entre 0,81 (DSTs) e 0,90 (Assertividade).

Visto a qualidade métrica da medida, ela tem ganhado notoriedade em âmbito internacional, dando contribuições importantes acerca desse construto e sua relação com outras variáveis. No contexto norteamericano, por exemplo, em uma pesquisa realizada por Parent e Moradi (2015), observou-se que a vergonha do próprio corpo foi associada à baixa autoeficácia no uso de preservativo. Por outro lado, a aceitação da sexualidade foi diretamente associada com maior uso de preservativos. Um outro achado interessante foi relatado por Davis et al. (2014) a partir de um estudo experimental. Tais pesquisadores observaram que o uso de bebida alcoólica diminuiu diretamente as intenções das mulheres heterossexuais de usarem preservativos no futuro. As mulheres com maior autoeficácia no uso de preservativos tinham intenções mais fortes de se envolverem na negociação com o parceiro para utilização de preservativos, no entanto, esse efeito foi moderado pela intoxicação com o álcool.

Destaca-se, entretanto, que apesar da importância do construto investigado e das vantagens que essa medida têm apresentado para fins de pesquisa, não foram encontradas medidas para avaliar a autoeficácia no uso de preservativos no Brasil. Assim, somada essa necessidade à grande incidência de casos de HIV e redução do uso do preservativo, o objetivo desta pesquisa foi adaptar essa medida para o contexto brasileiro por meio de dois estudos. O Estudo 1 que teve como objetivo principal dispor de uma versão brasileira da Escala de Autoeficácia no Uso 
de Preservativos (EAUP), realizando sua tradução e conferindo evidências de validade de construto e consistência interna; e o Estudo 2, o qual pretendeu replicar o modelo em uma amostra independente, comprovando o ajuste da estrutura multifatorial desta medida.

\section{Método}

\section{Participantes}

Contou-se com duas amostras não probabilísticas (por conveniência). A primeira, utilizada no Estudo 1 foi composta por 332 participantes das regiões Nordeste $(71,4 \%)$, Sudeste $(5,4 \%)$, Centro-oeste $(13,3 \%)$, Norte $(4,8 \%)$ e $\operatorname{Sul}(5,1 \%)$; sendo a maioria mulheres $(64 \%)$, com idades entre 18 e 60 anos $(\mathrm{M}=25,2 ; \mathrm{DP}=7,4)$, predominantemente jovens. Entre os participantes, $89,2 \%$ declararam-se heterossexuais e solteiros (69\%). Quanto ao uso de preservativos, 33,1\% afirmaram usar sempre, $28,3 \%$ frequentemente, $21,1 \%$ às vezes, $13,7 \%$ raramente e $3,9 \%$ nunca usaram. A maior parte da amostra era de universitarios $(59,6 \%)$ e $31,3 \%$ com renda familiar entre 1 a 3 salários mínimos. Já amostra utilizada no Estudo 2 contou com 907 participantes das cinco regiões do Brasil: Nordeste (24,5\%), Sudeste $(32,3 \%)$, Centro-oeste $(16,8 \%)$, Norte $(10,1 \%)$ e Sul $(15,5 \%)$, cujas idades variaram entre 18 e 55 anos $(\mathrm{M}=23,6 ; \mathrm{DP}=6,1)$. A maioria era do sexo feminino $(66,4 \%)$, heterossexuais $(86 \%)$, solteiros $(75,1 \%)$, com ensino superior incompleto $(72,5 \%)$, e possuíam renda de 1 a 3 salários mínimos $(25,7 \%)$. Em relação ao uso do preservativo, $37,3 \%$ admitiram usar sempre, $25,1 \%$ frequentemente, $16 \%$ às vezes, $19,1 \%$ raramente $\mathrm{e}$ $2,5 \%$ nunca usaram. O tamanho amostral se justifica pelo ponto de corte estabelecido para a carga fatorial mínima exigida na análise fatorial, que foi de 0,40 , a qual requer mais de 300 participantes (Guadagnoli \& Velicer, 1988). Como critério de inclusão dos respondentes considerou-se ter idade igual ou superior a 18 anos.

\section{Instrumentos}

Nos dois estudos foi utilizado um questionário composto por duas partes:

1. Escala de Autoeficácia no Uso de Preservativos (EAUP) - Adaptada por Asante e Doku (2010) em Gana, a escala avalia a autoeficácia no uso de preservativo, ou seja, a percepção do indivíduo em usar preservativos. Essa escala possui 14 itens distribuídos em uma escala Likert com os seguintes extremos: $1=$ "Discordo Totalmente" e $5=$ "Concordo Totalmente". O conjunto de itens se divide em quatro subescalas: Habilidade (colocar um preservativo em si mesmo ou no outro); a Assertividade (capacidade de convencer um(a) parceiro(a) a usar preservativo); Prazer e drogas (capacidade de usar preservativos quando sob a influência de alguma substância); e Doenças sexualmente transmissíveis - DSTs (receio de que o(a) parceiro(a) pense que tem uma doença sexualmente transmissível).

2. Questionário Sociodemográfico - Foram perguntados aos sujeitos questões como a cidade e estado onde residiam, além do sexo, idade, estado civil, nível de escolaridade, orientação sexual, renda familiar e frequência do uso de preservativo.

\section{Procedimento}

Os pesquisadores seguiram o preconizado na Resolução 510/16 do Conselho Nacional de Saúde, respeitando a autonomia dos participantes da pesquisa. Incialmente os itens foram traduzidos e retrotraduzidos de forma independente por dois psicólogos bilíngues. Em seguida, foi realizada uma comparação minunciosa das duas versões. No caso de traduções de itens não consensuais, um terceiro psicológico bilíngue opinou a fim de se obter conformidade. Após aprovação do comitê de ética em pesquisa (CAAE: 17887013.1.0000.5183), realizou-se a validação semântica do instrumento com 12 estudantes universitários no primeiro ano de curso, os quais demostraram adequada compreensão dos 14 itens. A aplicação dos questionários se deu por meio de um formulário online no site de domínio público Google Docs, enviado por meio de e-mail e redes sociais. Para ter um maior controle das respostas, utilizou-se a opção "permitir apenas uma resposta por pessoa". O formulário não permitia o envio de respostas com itens em branco (missing values). Além disso, questionários sem variabilidade de respostas foram desconsiderados uma vez que levantaram suspeita da inconsistência das respostas. Demandou-se a reposta individual dos participantes, que foram orientados a não se identificarem no questionário, assegurando o anonimato de sua participação. Foi indicado que poderiam deixar o estudo a qualquer momento sem penalização, garantindo o caráter voluntário. Todos assinalaram concordância no Termo de Consentimento Livre e Esclarecido apresentado previamente.

\section{Análise de dados}

Os dados foram tabulados e analisados por meio do software IBM SPSS (versão 22). Foram realizadas estatísticas descritivas (medidas de tendência central e dispersão) visando a caracterização da amostra. No Estudo 1 a análise dos componentes principais (Principal Components) foi efetuada a fim de conhecer a dimensionalidade da escala. Utilizou-se também 
da Análise Paralela (Hayton, Allen, \& Scarpello, 2004) para confirmar a quantidade de fatores que seriam extraídos. Posteriormente, verificou-se a consistência interna por meio do coeficiente alfa de Cronbach $(\alpha)$.

No Estudo 2 utilizou-se o AMOS (Analysis Moment Strutures, versão 21) com a finalidade de reunir evidências de ajuste do modelo fatorial da EAUP por meio de uma Análise Fatorial Confirmatória (AFC), considerando como entrada a matriz de covariâncias e o estimador de ML (Maximum Likelihood). A adequação de ajuste do modelo aos dados empíricos foi avaliada com os seguintes indicadores:

Razão Qui-quadrado/graus de liberdade - Esta é uma medida geral do ajuste do modelo (Byrne, 2010). Uma regra geral é que o modelo é adequado quando a razão do Qui-quadrado sobre os graus de liberdade $\left(\chi^{2} /\right.$ g.l.) for inferior a 1,96 (Ullman, 2007). Ressalta-se que o referido teste é sensível ao tamanho da amostra, levando à rejeição do modelo no caso de amostras grandes (Byrne, 2010).

Goodness-of-Fit Index (GFI) - Trata-se de um índice de qualidade do ajuste que descreve o quanto o modelo explica a proporção de variância-covariância dos dados, sendo considerados ideais os valores iguais ou superiores a 0,90 (Byrne, 2010; Garson, 2010; Hair, Black, Babin, Anderson, \& Tathan, 2009).

Comparative Fit Index (CFI) - É um índice de comparação de ajustamento de modelos; valores próximos a 0,90 ou superiores são admitidos como expressando um ajustamento adequado (Byrne, 2010; Garson, 2010; Hair et al., 2009).

Root-Mean-Square Error of Approximation (RMSEA) - É um índice comparativo de ajuste do modelo que leva em consideração o erro de aproximação na população e deve ser interpretado levando em consideração o intervalo de confiança de 90\% (IC90\%). Valores inferiores a 0,05 indicam um bom ajuste (Byrne, 2010). Alguns autores apontam, entretanto, que valores de RMSEA inferiores a 0,08 indicam um aceitável erro de aproximação (McDonald \& Ho, 2002; Thompson, 2000).

\section{Resultados}

Os resultados são descritos em subseções, de acordo com as análises psicométricas utilizadas. Primeiramente, no Estudo 1 apresentar-se-á a validade e consistência interna da EAUP por meio de Análise dos Componentes Principais (ACP) e os valores dos coeficientes alfa de Cronbach das dimensões encontradas. Posteriormente, o Estudo 2 apresenta os resultados da Análise Fatorial Confirmatória (AFC) em uma amostra maior de respondentes, por meio do método de equações estruturais.

\section{ESTUDO 1 \\ Validade de Construto e Consistência Interna}

Para testar a possibilidade da realização de uma ACP, inicialmente, verificou-se a fatorabilidade da matriz de correlações do instrumento. Utilizou-se do Kaiser-Meyer-Olkin (KMO), cujo índice utiliza as correlações parciais das variáveis, sendo aceitos valores iguais ou superiores a 0,60 ; e o Teste de Esfericidade de Bartlett que comprova a hipótese de que a matriz de covariâncias é uma matriz de identidade. Tal hipótese é rejeitada quando os valores são significativos. Os resultados obtidos indicaram a viabilidade deste tipo de análise estatística, com $\mathrm{KMO}=0,76$ e o Teste de Esfericidade de Bartlett significativo $\left[\mathrm{X}^{2}(91)=2108,992 ; \mathrm{p}<0,001\right]$.

$\mathrm{Na}$ sequência, para investigar a quantidade de componentes a ser extraído na ACP, utilizou-se da Análise Paralela (AP). Esta análise compara os valores próprios empíricos com aqueles obtidos paralelamente por meio de matrizes randômicas não correlacionadas, com a quantidade da amostra e número de variáveis iguais aos utilizados no estudo (Hayton, Allen, \& Scarpello, 2004). Ela propõe que os dados empíricos devem explicar mais variância do que aqueles gerados aleatoriamente. Os resultados mostraram que os valores próprios encontrados nas quatro primeiras dimensões empíricas foram maiores do que aqueles gerados aleatoriamente pela AP. Assim pode-se afirmar que o conjunto de itens possui quatro dimensões, corroborando com a literatura (Asante \& Doku, 2010).

Depois de encontrar o número de componentes a serem extraídos, buscou-se a realização de uma $\mathrm{ACP}$, fixando o número de fatores em 4 e levando em consideração carga fatorial de 0,40 (Pasquali, 2010). Todos os itens distribuíram-se com cargas fatoriais nos fatores propostos pela teoria. Os resultados podem ser visualizados na Tabela 1.

Como se pode-se observar na Tabela $\mathbf{1}$, todos os itens saturaram de maneira satisfatória nos seus respectivos componentes com cargas variando entre 0,47 e 0,91 . Tais componentes apresentaram valores próprios variando de 1,24 a 4,33, e explicando conjuntamente $66,8 \%$ da variância total. Ainda, com a finalidade de verificar a consistência interna da escala, utilizou-se o coeficiente alfa de Cronbach. Os componentes apresentaram coeficientes que variaram entre 0,60 e 0,90 . Os resultados são detalhados na Tabela 1. 
TABELA 1

Estrutura Fatorial da Escala de Auto-Eficácia no Uso de Preservativos.

\begin{tabular}{|c|c|c|c|c|}
\hline \multirow{2}{*}{ Itens } & \multicolumn{4}{|c|}{ Cargas } \\
\hline & $F 1$ & $F 2$ & $F 3$ & F4 \\
\hline $\begin{array}{l}\text { 02. Sinto-me confiante de que poderia colocar ou remover tranquilamente um } \\
\text { preservativo quando tenho relações. }\end{array}$ & 0,77 & 0,07 & 0,10 & $-0,06$ \\
\hline $\begin{array}{l}\text { 03. Sinto-me confiante em minha capacidade de colocar um preservativo em mim } \\
\text { ou em meu/minha parceiro(a) durante as preliminares. }\end{array}$ & 0,74 & 0,11 & 0,15 & $-0,06$ \\
\hline $\begin{array}{l}\text { 01. Me sentiria envergonhado(a) em botar um preservativo em mim } \\
\text { ou em meu/minha parceiro(a).* }\end{array}$ & 0,70 & 0,15 & 0,23 & $-0,07$ \\
\hline 05. Sinto-me confiante de que posso usar um preservativo com sucesso. & 0,66 & 0,03 & 0,06 & 0,26 \\
\hline $\begin{array}{l}\text { 04. Sinto-me confiante de que posso usar um preservativo com meu/minha } \\
\text { parceiro(a) sem "atrapalhar o momento". }\end{array}$ & 0,60 & $-0,00$ & 0,15 & 0,34 \\
\hline $\begin{array}{l}\text { 13. Não me sentiria confiante sugerindo o uso de preservativo a um(a) novo(a) } \\
\text { parceiro(a), porque sentiria medo dele(a) pensar que tenho uma doença } \\
\text { sexualmente transmissível.* }\end{array}$ & 0,09 & 0,91 & 0,24 & 0,09 \\
\hline $\begin{array}{l}\text { 14. Não me sentiria confiante sugerindo o uso de preservativo a um(a) novo(a) } \\
\text { parceiro(a), porque sentiria medo dele(a) pensar que já tive uma doença } \\
\text { sexualmente transmissível.* }\end{array}$ & 0,17 & 0,89 & 0,20 & 0,08 \\
\hline $\begin{array}{l}\text { 12. Não me sentiria confiante sugerindo o uso de preservativo a um(a) novo(a) } \\
\text { parceiro(a), porque sentiria medo dele(a) pensar que já tive experiências } \\
\text { homossexuais.* }\end{array}$ & 0,03 & $\mathbf{0 , 8 8}$ & 0,01 & $-0,06$ \\
\hline $\begin{array}{l}\text { 07. Sinto-me confiante em minha capacidade de sugerir o uso de preservativo } \\
\text { com um(a) novo(a) parceiro(a). }\end{array}$ & 0,25 & 0,16 & 0,86 & 0,11 \\
\hline $\begin{array}{l}\text { 06. Sinto-me confiante em minha capacidade para discutir o uso do preservativo } \\
\text { com qualquer parceiro(a) que eu possa ter. }\end{array}$ & 0,19 & 0,11 & 0,86 & 0,01 \\
\hline $\begin{array}{l}\text { 08. Sinto-me confiante de que poderia sugerir o uso de preservativo } \\
\text { sem que meu/minha parceiro(a) se sinta "doente". }\end{array}$ & 0,13 & 0,15 & 0,72 & 0,26 \\
\hline $\begin{array}{l}\text { 11. Sinto-me confiante de que posso lembrar do uso do preservativo mesmo } \\
\text { após ter usado drogas. }\end{array}$ & $-0,02$ & 0,06 & 0,04 & 0,84 \\
\hline $\begin{array}{l}\text { 10. Sinto-me confiante de que posso lembrar do uso do preservativo mesmo } \\
\text { após ter ingerido bebidas alcoólicas. }\end{array}$ & $-0,01$ & 0,10 & 0,17 & $\mathbf{0 , 8 1}$ \\
\hline $\begin{array}{l}\text { 09. Sinto-me confiante de que posso utilizar um preservativo durante uma } \\
\text { relação sem diminuir o prazer sexual. }\end{array}$ & 0,33 & $-0,13$ & 0,10 & 0,47 \\
\hline Valor Próprio & 4,33 & 2,09 & 1,70 & 1,22 \\
\hline Variância Total Explicada (\%) & 66,8 & & & \\
\hline Alfa de Cronbach & 0,77 & 0,90 & 0,82 & 0,60 \\
\hline
\end{tabular}

* Item invertido.

F1: Habilidade; F2: Doenças sexualmente transmissíveis; F3: Assertividade; F4: Prazer e drogas.

\section{ESTUDO 2 Comprovação da Estrutura Fatorial}

Baseado na teoria de Brafford e Beck (1991) e coerente com o modelo fatorial proposto pelo estudo de Asante e Doku (2010), testou-se o modelo tetradimensional dos 14 itens da EAUP. Assim, por meio de uma AFC foram observados os seguintes indicadores de ajuste: $\chi^{2} / \mathrm{g} .1 .=6,79 ; \mathrm{GFI}=0,93$; $\mathrm{CFI}=0,91 ; \quad \mathrm{RMSEA}=0,08 \quad(\mathrm{IC} 90 \%=0,07-0,09)$. Observa-se que tanto o GFI quanto o CFI apresentaram valores acima de 0,90 . Tais índices são considerados bons pela literatura (Byrne, 2010; Garson, 2010; Hair, Black, Babin, Anderson, \& Tathan, 2009). Além disso, o RMSEA, ponderando seu intervalo de confiança apresentou o limite mínimo aceitável. Quanto ao $\chi^{2} / \mathrm{g} .1$, deve-se destacar que apesar de ter apresentado valores aquém do estipulado, este é influenciado pelo tamanho da amostra. Assim, nesse caso em específico, ele poderá ser desconsiderado. Os valores aqui encontrados satisfazem os índices estabelecidos pela literatura, confirmando dessa forma o ajuste do modelo. A estrutura fatorial da EAUP pode ser observada na Figura 1. 


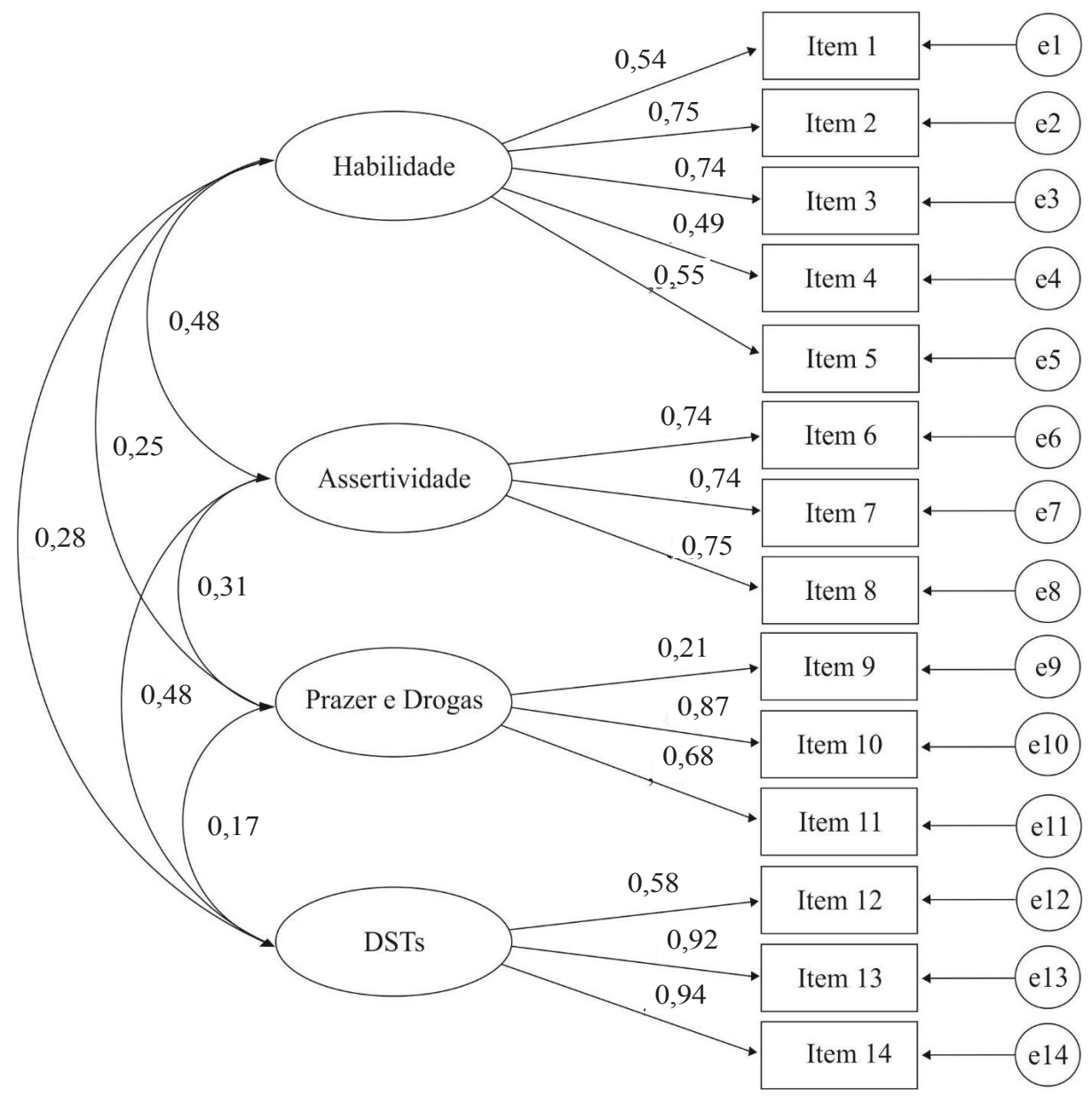

Figura 1. Estrutura fatorial da escala de autoeficácia no uso de preservativos

Como se pode perceber todos os pesos fatoriais (Lambdas $-\lambda$ ) foram positivos e estatisticamente diferentes de zero $(0 ; t>1,96, p<0,001)$ com valores entre 0,21 e 0,94 . Além disso, as pontuações brutas desses quatro componentes apresentaram correlações entre si que variaram entre 0,17 e 0,48 ; ambas estatisticamente significativas $(p<0,05)$, indicando que são fatores moderadamente correlacionados, porém dependentes. Tal aspecto é bastante comum, visto que as dimensões medem o mesmo construto.

\section{Discussão}

Avaliar a autoeficácia no uso de preservativos demanda a existência de meios que possibilitem conhecer o construto avaliado por meio de múltiplas observações, o que pode ser alcançado levando-se em consideração escalas psicométricas (Pasquali, 2010). $\mathrm{O}$ objetivo desta pesquisa foi adaptar para o contexto brasileiro a EAUP, que é a medida de autoeficácia mais comumente utilizada para avaliar alterações comportamentais na prevenção das ISTs em jovens (Santos et al., 2017). Para isso buscou-se reunir evidências de sua validade de construto, por meio da verificação de sua validade fatorial e consistência interna. Com base nos resultados apresentados, os quais corroboraram o modelo teórico proposto por Asante e Doku (2010), acredita-se que esse objetivo tenha sido alcançado.

Em relação às análises fatoriais, destaca-se que esta pesquisa foi além da proposta de Asante e Doku (2010), os quais empregaram apenas análises de natureza exploratória. Isso porque, ao contrário do estudo desses autores, esta pesquisa também contou com análises fatoriais confirmatórias. Os resultados apontaram que a EAUP aplicada à amostra brasileira apresentou-se de forma coerente com o modelo proposto para a sua versão com 14 itens e a extração de quatro componentes, incialmente comprovadas pela Análise dos Componentes Principais.

A partir dos estudos prévios foi corroborada a distribuição dos componentes: Habilidade, Asser- 
tividade, Prazer e Drogas e Doenças sexualmente transmissíveis, sugerindo bons índices de validade de construto e de confiabilidade da medida. No segundo estudo, a solução tetrafatorial foi testada, e adequados indicadores de ajuste foram verificados por meio da AFC: $\chi^{2} / \mathrm{g} .1 .=6,79 ; \mathrm{GFI}=0,93 ; \mathrm{CFI}=0,91$; RMSEA $=0,08$ (IC90\% $=0,07-0,09)$, conforme os propostos por Byrne (2010). Enfatiza-se que a razão qui-quadrado pelos graus de liberdade é sensível ao tamanho da amostra, apresentando-se superior ao esperado. Considerando que os principais índices de ajuste do modelo foram satisfatórios sem a necessidade de correlacionar erros, preferiu-se apresentar uma estrutura sem modificações.

Também se faz importante compreender os fatores identificados, considerando a cultura brasileira. $\mathrm{O}$ primeiro fator foi composto por cinco itens e identificado como "Habilidade". Tal fator está relacionado com a apropriação e habilidade mecânica de uso de preservativos (e.g. "Sinto-me confiante de que poderia colocar ou remover tranquilamente um preservativo quando tenho relações"). O segundo fator foi composto por três itens e identificado como "Doenças sexualmente transmissíveis". Os itens deste fator expressam estigmas associados às doenças sexualmente transmissíveis e ao receio do(a) parceiro(a) não aprovar o pedido de uso de preservativos (e.g. "Não me sentiria confiante sugerindo o uso de preservativo a um(a) novo(a) parceiro(a), porque sentiria medo dele(a) pensar que tenho uma doença sexualmente transmissível"). O terceiro fator, igualmente composto por três itens, foi identificado como "Assertivividade". Similarmente ao estudo de Asante e Doku (2010), os itens saturados neste fator estão relacionados às dimensões de autoeficácia da assertividade, das habilidades de negociação e da capacidade de persuadir um(a) parceiro(a) a usar preservativo. Por fim, o quarto fator composto por três itens foi identificado como "prazer e drogas" (originalmente Pleasure and Intoxicants). Tal fator é indicativo da capacidade de usar preservativos sob a influência de álcool ou drogas sem sentir uma redução na sensação sexual (e.g. "Sinto-me confiante de que posso lembrar do uso do preservativo mesmo após ter usado drogas").

Embora tais achados tenham sido coerentes com àqueles apresentados por Asante e Doku (2010), apontando uma estrutura dimensional e composição exatamente igual à encontrada por esses autores, os resultados não foram de todo surpreendentes. Os valores alfas aqui indicados foram inferiores àqueles encontrados no estudo desses autores no qual todas as dimensões apresentaram alfas acima de 0,70.
Além disso, a estrutura encontrada foi diferente da apresentada em outros estudos, os quais mostraram arranjos diferentes, seja de número de fatores seja de composição ou consistência dos itens em cada fator (Barkley \& Burns, 2000; Shaweno \& Tekletsadik, 2013). Tal fato, entretanto, já era esperado devido a sensibilidade da medida para diferenças culturais (Asante \& Doku, 2010).

Quanto à consistência interna, o fato de nesta pesquisa, apenas algumas dimensões terem apresentado alfas superior a esse ponto de corte, sugere que o uso dessa medida deve ser restrito à pesquisa, não podendo o mesmo ser empregado para propósitos clínicos. Tal fato não desmerece os resultados aqui encontrados, já que a qualidade teórica e psicométrica dessa medida tem permitido sua utilização em pesquisa em uma diversidade de contextos, tendo encontrado informações muito importantes a respeito dos comportamentos de uso de preservativo. Isso pode ser evidenciado em várias pesquisas recentes como, por exemplo, o estudo realizado por Asante, Osafo e Doku (2016). Tais autores, utilizando-se de regressão hierárquica, encontraram dados que suportam que as dimensões dessa medida predizem tanto as intenções de uso do preservativo no futuro, quanto o relato de uso na última relação sexual.

O conhecimento obtido nessas pesquisas com o auxílio da EAUP é de especial relevância na medida que torna possível pensar em estratégias educativas de saúde voltadas para grupos específicos. As mulheres, por exemplo, apresentam padrões de comportamento diferentes quando comparados aos homens, frente a questões relacionadas ao sexo, como pode ser observado em um estudo realizado na África do Sul por Matseke, Peltzer, Mchunu, e Louw, (2012). Nessa pesquisa foi observada uma menor porcentagem de uso de preservativo para esse grupo quando comparadas aos homens. Os autores inferiram que os resultados poderiam ser explicados por questões relacionadas a normas de comportamento sexual enraizadas na cultura local, que admitem que apenas os homens tomem decisões relacionadas ao sexo. Nesse caso, especificamente, a EUAP poderia ser usada para identificar quais as dimensões que estariam mais contribuindo para esse comportamento em ambos sexos. Por meio da identificação das dimensões mais afetadas seria possível traçar um plano de estratégia específico no domínio mais penalizado. Segundo Bandura, a autoeficácia é um dos principais mecanismos pelos quais se exerce a ação humana. A percepção da autoeficácia determinará o tipo de comportamento escolhido, a quantidade de esforço dedicado a enfrentar situações 
difícieis e o tempo de persistência na tentativa de solucioná-los (Álvaro \& Garrido, 2006). Assim, dada a relevância da autoeficácia, espera-se que pesquisas futuras abordem tal construto considerando o comportamento sexual no contexto brasileiro.

Além disso, identificar as dimensões de autoeficácia afetadas pode ajudar a entender melhor o problema, já que como lembram Brum e Carrara (2012), o uso da camisinha trata-se de um comportamento social complexo. Os autores afirmam que devido a essa complexidade, a resolução desse problema deveria passar por ações de políticas públicas que estabeleçam metacontingências claras, que descrevam basicamente quais os comportamentos esperados, onde e como eles podem ou devem ocorrer e quais seriam as consequências imediatas e de longo prazo no que diz respeito à saúde dos envolvidos. Os autores defendem, ainda, a inclusão de um trabalho interdisciplinar e intersetorial, envolvendo escolas, postos de saúde, associações comunitárias, dentre outros setores.

Assim, dada essa complexidade e variação da autoeficácia por aspectos externos, como variação cultural, econômica e social, além de características intrínsecas, como personalidade, valores e atitudes, demanda-se o emprego dessa medida em pesquisas correlacionais. Espera-se que tais pesquisas busquem relacionar as dimensões da EAUP com essas diversas variáveis para verificar como elas afetam os comportamentos de autoeficácia no uso do preservativo. De igual importância também seria interessante testar os efeitos moderadores e mediadores do construto autoeficácia, com a finalidade de melhor explicar o construto no contexto brasileiro.

Desde o surgimento da epidemia da aids no cenário mundial, a prevenção da transmissão do HIV tem sido um dos maiores desafios no controle da doença (Gubert, Vieira, Damasceno, Lima, \& Ximenes, 2010). Assim, a utilização de medidas de autoeficácia no uso de preservativos pode, ainda, esclarecer as razões para a não utilização do preservativo entre jovens e adultos de ambos os sexos no contexto nacional. Tal conhecimento pode subsidiar políticas públicas e o desenvolvimento de competências relacionadas com o uso seguro do preservativo. Em estudos posteriores é importante conhecer possíveis relações da autoeficácia no uso de preservativos com outros construtos, tais como personalidade, autoestima, imagem corporal, gênero e identidade étnica ou cultural. Estas relações são essenciais para compreender o fenômeno e para agir sobre o processo prevenção-doença.
Também seria interessante o aperfeiçoamento da medida em estudos nacionais, testando, por exemplo, a validade convergente e discriminante da EAUP com outras medidas de comportamento relacionados ao uso de preservativos. Além disso, demanda-se testar a invariância fatorial quanto a algumas variáveis como orientação sexual, sexo e idade, ou mesmo os parâmetros dos seus itens por meio de modelos da Teoria de Reposta ao Item (TRI). O International Test Commission também aponta para a necessidade de estudos de funcionamento diferencial dos itens DIF (differential item functioning analysis) quando é realizada a tradução e adaptação de instrumentos para outros contextos. Considera-se que o processo de adaptação da EAUP aqui realizado indica a capacidade dos itens para representar os conceitos e domínios da escala na nova população-alvo (Borsa, Damásio \& Bandeira, 2012; ITC, 2017). Ressalta-se, no entanto, que novos estudos psicométricos brasileiros devem ser realizados a fim de obter evidências de validade considerando outras amostras e contextos.

É importante destacar que a presente pesquisa não está isenta de limitações. Especificamente, tratouse de amostras em que a maioria dos respondentes possuía ensino superior incompleto, com rendas características prováveis de classe média e direcionada a universitários. Além disso, as duas amostras foram de conveniência. Contou-se com a participação daqueles que concordaram em colaborar para a pesquisa de forma não aleatória, devendo-se, por isso, ponderar a generalização dos resultados.

\section{Considerações finais}

Os resultados sugerem que a versão brasileira da EAUP, proposta nesta pesquisa, mostra-se adequada para avaliar a autoeficácia no uso de preservativo considerando suas dimensões de habilidades de negociação, capacidade de utilizar preservativos sob a influência de álcool ou drogas e o medo ou receio vinculados às ISTs. Tais dimensões se configuram como legítimas, sendo comprovadas tanto no primeiro quanto no segundo estudo desta pesquisa.

Confia-se, assim, estar oferecendo uma medida psicometricamente adequada e de fácil aplicação para uso em pesquisas no âmbito da saúde. Ainda, após estudos de aprimoramento da escala, esta pode ser também aplicada ao domínio das políticas públicas, onde seja importante fornecer informações a respeito do nível de autoeficácia de determinado grupo ou acerca das dificuldades e necessidades reais da população em diferentes contextos. Isso é de fundamental importância, visto a natureza complexa do problema. 


\section{Referências}

Álvaro, J. L. \& Garrido, A. (2006). Psicologia social-perspectivas psicológicas e sociológicas. São Paulo: McGrawHill.

Asante, K. O. \& Doku, P. N. (2010). Cultural adaptation of the condom use self efficacy scale (CUSES) in Ghana. BMC public health, 10(1), 227. 10.1186/1471-2458-10-227

Asante, K. O., Osafo, J., \& Doku, P. N. (2016). The Role of Condom Use Self-Efficacy on Intended and Actual Condom Use Among University Students in Ghana. Journal of Community Health, 41, 97-104. 10.1007/s10900-015-0073-6

Bandura, A. (1986). Social Foundations of Thought and Actions: A Social Cognitive Theory. Prent ice-Hall, Englewood Cliffs: New Jersey.

Bandura, A. (1994). Social cognitive theory and exercise of control over HIV infection. In Preventing AIDS. Springer US.

Barkley, T. W. \& Burns, J. L. (2000). Factor analysis of the Condom Use Self-efficacy Scale among multicultural college students. Health Education Research, 15(4), 485-489. 10.1093/her/15.4.485

Berquó, E., Barbosa, R. M., \& Lima, L. P. (2008). Uso do preservativo: tendências entre 1998 e 2005 na população brasileira. Revista de Saúde Pública, 42 (suppl. 1), 34-44. 10.1590/S0034-89102008000800006

Borsa, J. C., Damásio, B. F., \& Bandeira, D. R. (2012). Adaptação e validação de instrumentos psicológicos entre culturas: Algumas considerações. Paidéia (Ribeirão Preto), 22(53), 423-432. 10.1590/1982-43272253201314

Brafford, L. J. \& Beck, K. H. (1991). Development and validation of a condom self-efficacy scale for college students. Journal of American College Health, 39(5), 219-225. 10.1080/07448481.1991.9936238

Brien, T. M., Thombs, D. L., Mahoney, C. A., \& Wallnau, L. (1994). Dimensions of self-efficacy among three distinct groups of condom users. Journal of American College Health, 42, 167-174. abs/10.1080/07448481.1994.9939665.

Byrne, B. M. (2010). Structural equation modeling with Amos: basic concepts, applications, and programming (2 $\left.2^{\mathrm{a}} \mathrm{ed}.\right)$. Ottawa: Routledge.

Brum, M. M., Carrara, K. (2012). História individual e práticas culturais: efeitos no uso de preservativos por adolescentes. Estudos de Psicologia (Campinas), 29(supl. 1), 689-697. 10.1590/S0103-166X2012000500005

Davis, K. C., Masters, N. T., Eakins, D., Danube, C. L., George, W. H., Norris, J., \& Heiman, J. R. (2014). Alcohol intoxication and condom use self-efficacy effects on women's condom use intentions. Addictive Behaviors, 39, 153158. 10.1016/j.addbeh.2013.09.019

Dourado, M. I. C., MacCarthy, S., Reddy, M., Calazans, G., \& Gruskin, S. (2015). Revisitando o uso do preservativo no Brasil. Revista Brasileira de Epidemiologia, 18 (suppl. 1), 63-88. 10.1590/1809-4503201500050006

Cohen, S. E., Liu, A. Y., Bernstein, K. T., \& Philip, S. (2013). Preparing for HIV pre-exposure prophylaxis: lessons learned from post-exposure prophylaxis. American journal of preventive medicine, 44(102), S80-S85. 10.1016/j. amepre.2012.09.036

Garson, G. D. (2010). PA 765 Statnotes: an online textbook. Disponível em: <http://faculty.chass.ncsu.edu/garson/PA765/ statnote.htm> Acessado em: 07 ago. 2015.

Guadagnoli, E. \& Velicer, W. F. (1988). Relation of sample size to the stability of component patterns. Psychological Bulletin, 103, 265-275. 10.1037/0033-2909.103.2.265

Gubert, F. do A., Vieira, N. F. C., Damasceno, M. M. C., Lima, F. E. T., \& Ximenes, L. B. (2010). Escalas para medida de comportamento preventivo em meninas adolescentes frente às DST/HIV: revisão integrativa. Revista Gaúcha de Enfermagem, 31(4), 794-802. 10.1590/S1983-14472010000400025

Hair, J. F., Black, W. C., Babin, B. J., Anderson, R. E., \& Tatham, R. L. (2009). Análise multivariada de dados (6-a ed.). Porto Alegre: Bookman.

Hayton, J. C., Allen, D. G., \& Scarpello, V. (2004). Factor retention decisions in exploratory factor analysis: A tutorial on parallel analysis. Organizational research methods, 7(2), 191-205. 10.1177/1094428104263675

International Test Commission. (2017). The ITC Guidelines for Translating and Adapting Tests (Second edition). Recuperado em: 02 nov. 2017, de <https://www.intestcom.org/files/guideline_test_adaptation_2ed.pdf>.

International Test Commission (2010). International Test Commission guidelines for translating and adapting tests. Recuperado em 24 jul. 2012, de <http://www.intestcom.org/upload/sitefi les/40.pdf>.

Lima, M. D. S., Martins-Melo, F. R., Heukelbach, J., Alencar, C. H., Boigny, R. N., \& Ramos Júnior, A. N. (2016). Mortality related to tuberculosis-HIV/AIDS co-infection in Brazil, 2000-2011: epidemiological patterns and time trends. Cadernos de saúde publica, 32(10), 1-11. 10.1590/1414-462X2016000100x1

Longo, L. A. D. B. (2013). Juventude e contracepção: um estudo dos fatores que influenciam o comportamento contraceptivo das jovens brasileiras de 15 a 24 anos. Revista Brasileira de Estudos de População, 19(2), 229-247. Recuperado de <https://www.rebep.org.br/revista/article/viewFile/320/pdf_301>.

Matseke, G., Peltzer, K., Mchunu, G., \& Louw, J. (2012). Correlates of Condom Use among Male and Female aged 18-24 Years in South Africa. Gender \& Behaviour, 10(2), 4627 -4644. Recuperado de <https://www.researchgate. net/publication/291975212_Correlates_of_Condom_Use_among_Male_and_Female_aged_18-24_Years_in_South_ Africa>. 
Ministério da Saúde (2016). Boletim Epidemiológico - Aids e DST. Ministério da Saúde - Secretaria de Vigilância em Saúde - Departamento de DST, Aids e Hepatites Virais. Brasília, DF. Recuperado de: <http://www.aids.gov.br/sites/ default/files/anexos/publicacao/2016/59291/boletim_2016_1_pdf_16375.pdf>.

Myers, D. G. (2000). Psicologia Social (6 ${ }^{\underline{a}}$ ed.). Rio de Janeiro: LTC.

Parent, M. C. \& Moradi, B. (2015). Self-Objectification and Condom Use Self-Efficacy in Women University Students. Archives of Sexual Behavior, 44, 971-981. 10.1007/s10508-014-0384-1

Pasquali, L. (2010). Instrumentação psicológica: fundamentos e práticas. Porto Alegre: Artmed.

Pereira, B. S., Costa, M. C. O., Amaral, M. T. R., da Costa, H. S., da Silva, C. A. L., \& Sampaio, V. S. (2014). Fatores associados à infecção pelo HIV/AIDS entre adolescentes e adultos jovens matriculados em Centro de Testagem e Aconselhamento no Estado da Bahia, Brasil. Revista Ciência \& Saúde Coletiva, 19(3), 747-758. 10.1590/141381232014193.16042013

Pinheiro, T. F., Calazans, G. J., \& Ayres, J. R. D. C. M. (2013). Uso de Camisinha no Brasil: um olhar sobre a produção acadêmica acerca da prevenção de HIV/Aids (2007-2011). Temas em Psicologia, 21(3), 815-836. 10.9788/TP2013.3EE07PT

Ribeiro, K. C. S., Silva, J. D., \& Saldanha, A. A. W. (2011). Querer é poder? A ausência do uso de preservativo nos relatos de mulheres jovens. DST-Jornal Brasileiro de Doenças Sexualmente Transmissiveis, 23(2), 84-89. 10.5533/21778264-201123206

Santos, M. J., Ferreira, E., Duarte, J., \& Ferreira, M. (2017). Adaptação portuguesa e validação da versão reduzida da Condom Use Self-efficacy Scale. Revista Internacional de Andrología, 15(1), 23-30. 10.1016/j.androl.2016.06.002

Shaweno, D. \& Tekletsadik, E. (2013). Validation of the condom use self-efficacy scale in Ethiopia. BMC international health and human rights, 13(1), 22. 10.1186/1472-698x-13-22

Sousa, C. S. P., Martins, E. S., Menezes, A. V. B., Pereira, M. L. D., Cordeiro, L. I., Pinheiro, A. K. B., \& Barbosa, R. C. M. (2016). Evaluation of instruments for the use of a condom. International Archives of Medicine, 232(9), 1-8, $10.3823 / 2103$

Unaids (2016). Global Report: When Women Lead, Change Happens. Recuperado de: <http://www.unaids.org/sites/ default/files/media_asset/when-women-lead-change-happens_en.pdf $>$.

Thompson, B. (2000). Ten Command ments of structural equation modeling. In L. Grim \& G. P. Yarnold (Orgs.). Reading and understanding more multivariate statistics (pp. 261-284). Washington: APA.

Tronco, C. B. \& Dell'Aglio, D. D. (2012). Caracterização do comportamento sexual de adolescentes: iniciação sexual e gênero. Gerais: Revista Interinstitucional de Psicologia, 5(2), 254-269. Recuperado de: <http://pepsic.bvsalud.org/ pdf/gerais/v5n2/v5n2a06.pdf>.

Ullman, J. B. (2007). Structural Equation Modeling. In B. G. Tabachnick \& L. S. Fidell (Orgs.). Using multivariate statistics (5 $5^{\underline{a}}$ ed.). Boston: Pearson Education.

Wulfert, E., \& Wan, C. K. (1993). Condom use: a Self-efficacy Model. Health Psychology, 12(3), 346-353. http://dx.doi. org/10.1037/0278-6133.12.5.346

Dados dos autores:

Josemberg Moura de Andrade - Doutor, Universidade de Brasília.

Kaline da Silva Lima - Mestranda, Universidade Federal da Paraíba.

Valdiney Veloso Gouveia - Doutor, Universidade Federal da Paraíba.

Hemerson Fillipy Silva Sales - Doutorando, Universidade Federal da Paraíba

Elida de Farias Melo - Graduada, Universidade Federal da Paraíba.

Vivianne Freitas Oliveira Asfora - Mestre, Universidade Federal da Paraíba.

Endereço para correspondência:

Josemberg Moura de Andrade

SHIN QL 05, Conjunto 05, Casa 05 - Lago Norte

75505-755, Brasília, DF, Brasil

<Josemberg.andrade@gmail.com>

Recebido em: 08.08.2017

Aceito em: 02.01.2018 